\title{
Quelques Spirurides de Poissons de la région nantaise
}

\author{
par Annie J. PETTER \\ Laboratoire de Zoologie (Vers) associé au C.N.R.S., Muséum National d'Histoire Naturelle \\ ( $\mathrm{P}^{r}$ A.-G. Chabaud), 57, rue Cuvier - F 75 - Paris-5 \\ Laboratoire de Parasitologie et Pathologie exotique ( $\mathrm{P}^{\mathrm{r}} \mathrm{C}$. VermeIL), \\ et Laboratoire d'Anatomie pathologique ( $\mathrm{P}^{\mathrm{r}}$ J.-P. Kerners), Faculté de Médecine de Nantes, \\ 1, rue Gaston-Veil - 44 - Nantes.
}

\section{Résumé}

Nous décrivons plusieurs Spirurides adultes et larvaires récoltés chez des Poissons et Crustacés au Croisic (Bretagne) :

- le mâle et les larves de Spinitectö̈des berlandi Petter 1970 recueillis respectivement chez Molva molva (L.) et Gadus luscus L. L'aspect de l'extrémité caudale du mâle rapproche l'espèce du genre Ascarophis.

- Cristitectus congeri n. g., n. sp. recueilli dans l'estomac d'un Conger vulgaris Cuv.; l'espèce est caractérisée par la présence de courtes côtes cuticulaires disposées en cercle à l'extrémité antérieure. Nous interprétons cette espèce comme un Cystidicolinae primitif, à cause de la présence de pseudo-lèvres très développées et de 8 papilles au cycle externe.

- des femelles d'Ascarophis morrhuae van Bened., recueillies chez Gadus luscus L. Les œufs de ces femelles possèdent en plus des 2 filaments principaux à l'un des pôles, des filaments très fins en nombre variable à chacun des pôles.

- des larves d'Ascarophis morrhuae recueillies chez un Carcinus maenas Penn.

- un mâle d'Ascarophis sp. recueilli dans un crabe trouvé dans l'estomac d'un Trigla sp.

- des larves d'Ascarophis sp. recueillies à l'autopsie d'un Acanthocottus bubalis (Euphr.). Cette larve possède un pharynx court et des pseudo-lèvres très développées. 


\section{Summary}

Several Spirurids are described. They were collected from fishes and Crustacea at Le Croisic (Bretagne); they include the following adulte and larval stages:

- Males and larvae of Spinitectoïdes berlandi Petter 1970 respectively collected from Molva molva (L.) and Gadus luscus L. The male caudal extremity suggests a relationship to the genus Ascarophis.

- Cristitectus congeri n. g., n. sp. was collected from the stomach of Conger vulgaris Cuv.; the species is characterised by short cuticular ridges located on the anterior end. The author is regarding this species as a primitive Cystidicolinae on account of its highly-developed pseudo-lips and its eight papillae on the outer ring.

- Some females of Ascarophis morrhuae v. Ben. were collected from Gadus luscus L. On their eggs some very fine filaments were observed in different numbers on each poles in addition to the two main ones already present on a single pole.

- Some Ascarophis morrhuae larvae were collected from a Carcinus maenas Penn.

- A male of Ascarophis sp. was found in a crab collected from a Trigla sp. stomach.

- Some Ascarophis sp. larvae were collected from a dissected Acanthocottus bubalis (Euphr.). This larva has a short pharynx and strongly developed pseudo-lips.

Dans une note précédente (Petter 1970), nous décrivions un certain nombre de Nématodes de Poissons récoltés au cours d'un stage au Croisic (Bretagne) en juillet et août 1968 ; un second stage effectué en juillet 1969 nous a permis de compléter nos récoltes ; nous avons, en particulier, trouvé plusieurs Spirurides adultes et larvaires dont nous donnons la description ici :

\section{Spinitectoides berlandi Petter 1970 .}

Dans notre note précédente, nous décrivions une espèce dont nous avions trouvé uniquement trois femelles chez Molva molva (L.) et une larve chez Gadus luscus L; Berland avait déjà en 1961 décrit cette espèce uniquement sur les femelles. L'autopsie d'une nouvelle Molva molva nous a procuré de très nombreux spécimens de cette espèce dans l'intestin, dont plusieurs mâles dont nous donnons la description ; de plus, nous avons récolté dans un Gadus luscus de nombreuses larves, ce qui nous a permis d'effectuer une étude plus complète de celles-ci, et en particulier de leur structure apicale. 


\section{Description :}

MâLE : Les mâles mesurent environ $30 \mathrm{~mm}$; la région antérieure a le même aspect que chez la femelle; l'extrémité postérieure forme plusieurs tours de spire.

Principales mensurations d'un mâle long de $28 \mathrm{~mm}$; largeur maximum : $200 \mu$; pharynx : $90 \mu$; œsophage musculaire : $650 \mu$; œsophage glandulaire : $4.000 \mu$; deiri-

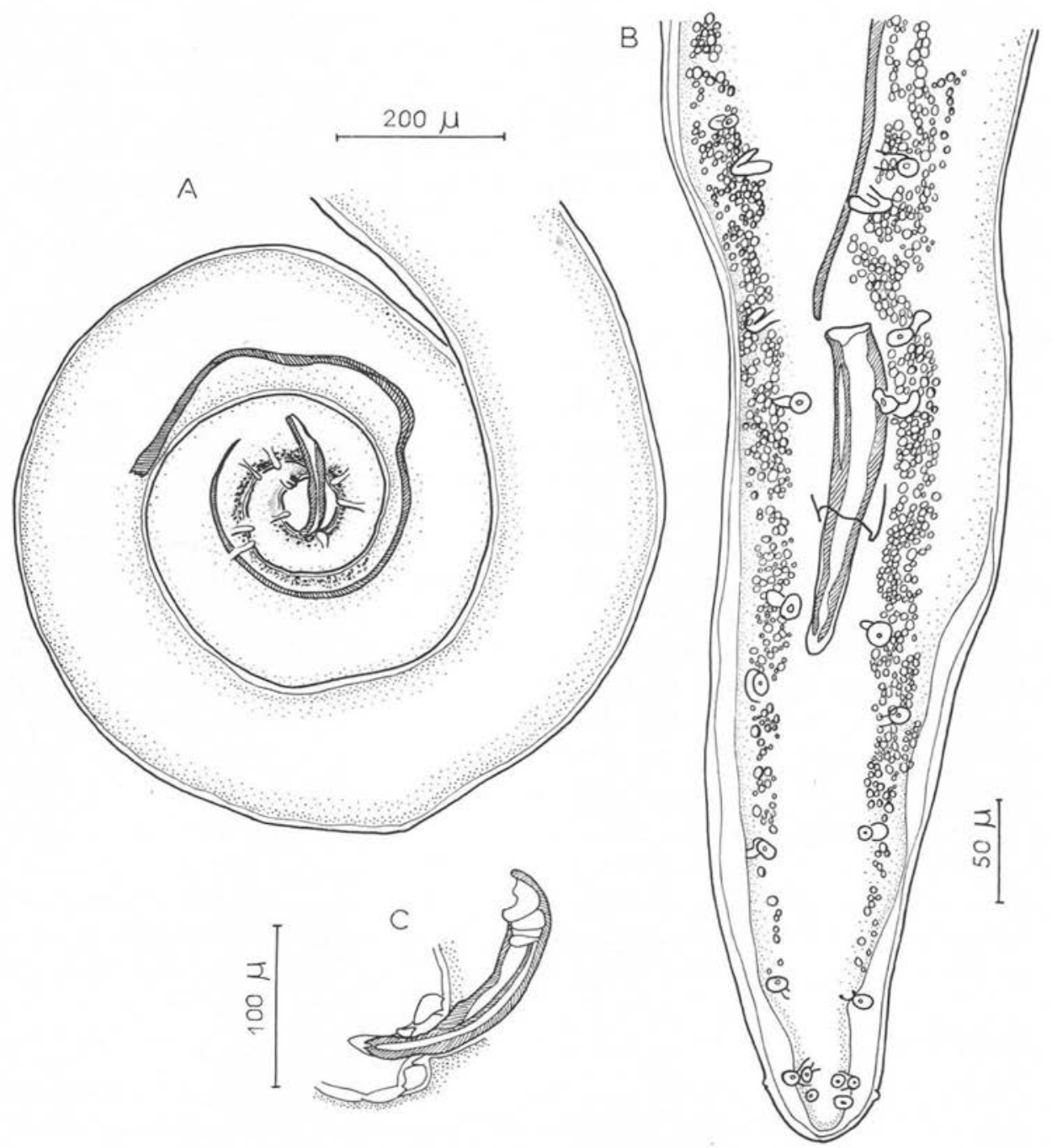

Fig. 1. - Spinitectoïdes berlandi Petter 1970, mâle; A: extrémité caudale, vue latérale ; B : extrémité caudale, vue ventrale; C: petit spicule 
des, anneau nerveux et pore excréteur situés respectivement à $80 \mu, 100 \mu$ et $140 \mu$ de l'extrémité antérieure ; queue : $300 \mu$; spicule gauche : $160 \mu$; spicule droit : $1.000 \mu$; longueur des ailes caudales : $350 \mu$.

Comme chez la femelle, la région antérieure est ornée de festons arrondis; le premier cercle de festons, très petits, débute à $110 \mu$ de l'extrémité antérieure ; les festons suivants sont beaucoup plus grands ; à environ $4.000 \mu$ de l'extrémité antérieure, l'ornementation s'atténue beaucoup et finit par disparaître complètement. Il existe d'étroites ailes caudales; on observe (fig. 1, A, B), 7 paires de papilles post-cloacales et 4 paires de papilles pré-cloacales; les papilles pré-cloacales comprennent un groupe terminal de trois paires de papilles et 4 paires de papilles disposées régulièrement le long de la queue; les phasmides sont à la hauteur du groupe terminal de papilles; on observe deux rangées latéro-ventrales de petites granulations disposées entre les papilles ; elles débutent à $80 \mu$ de l'extrémité postérieure et s'étendent sur une longueur de $500 \mu$.

Le grand spicule est très long et effilé ; le petit spicule est court et trapu. (fig. 1, C).

LARVE: Les larves trouvées chez Gadus luscus sont plus petites et plus fines que les adultes ; d'après l'aspect des ébauches génitales, il doit s'agir de larves du $4^{\circ}$ stade.

Principales mensurations d'une larve longue de $11 \mathrm{~mm}$ : largeur maximum: $120 \mu$; pharynx : $65 \mu$; œsophage musculaire : $450 \mu$; œsophage glandulaire : $1.920 \mu$; deirides, anneau nerveux et pore excréteur situés respectivement à $55 \mu, 70 \mu$ et $125 \mu$ de l'extrémité antérieure; queue : $120 \mu$; la première strie cuticulaire, dépourvue de festons, est située à $60 \mu$ de l'extrémité antérieure ; les stries suivantes portent des festons à bord postérieur droit et non arrondi comme chez l'adulte (fig. 2, A).

Comme chez l'adulte, l'ouverture buccale (fig. 2, B), est losangique et porte sur ses angles latéraux 2 languettes dressées; on observe également immédiatement sous l'ouverture buccale un cercle de dents internes, mais il comprend ici 2 dents latérales simples et 4 dents submédianes bicuspides et non comme chez l'adulte 14 dents simples : il existe 4 papilles submédianes et 2 amphides.

L'extrémité caudale se termine par un petit mucron arrondi (fig. 2, D).

L'ébauche génitale femelle est constituée par 2 longs ovaires opposés reliés par un long tube très fin, ébauche des voies génitales; l'ébauche vulvaire est située au milieu de ce tube, à $4,2 \mathrm{~mm}$ de l'extrémité postérieure. L'ébauche génitale mâle comprend un testicule (fig. 2, C) prolongé par un long tube fin qui est l'ébauche du canal déférent ; au niveau de l'anus, on observe une poche (fig. 2, E), qui est l'ébauche du cloaque.

\section{Diseussion :}

Dans notre note précédente, nous classions notre espèce dans les Cystidicolinae ; nous signalions que la capsule buccale presque circulaire de cette espèce évoquait les Rhabdochoninae, mais que nous attendions de connaître le mâle pour confirmer ou infirmer ses affinités avec cette sous-famille; nous voyons maintenant que l'extrémité 

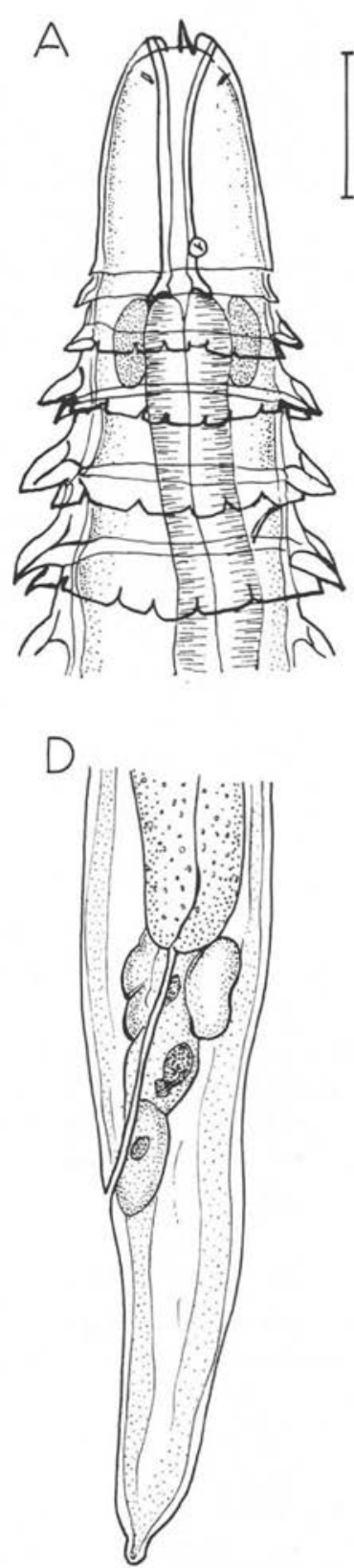
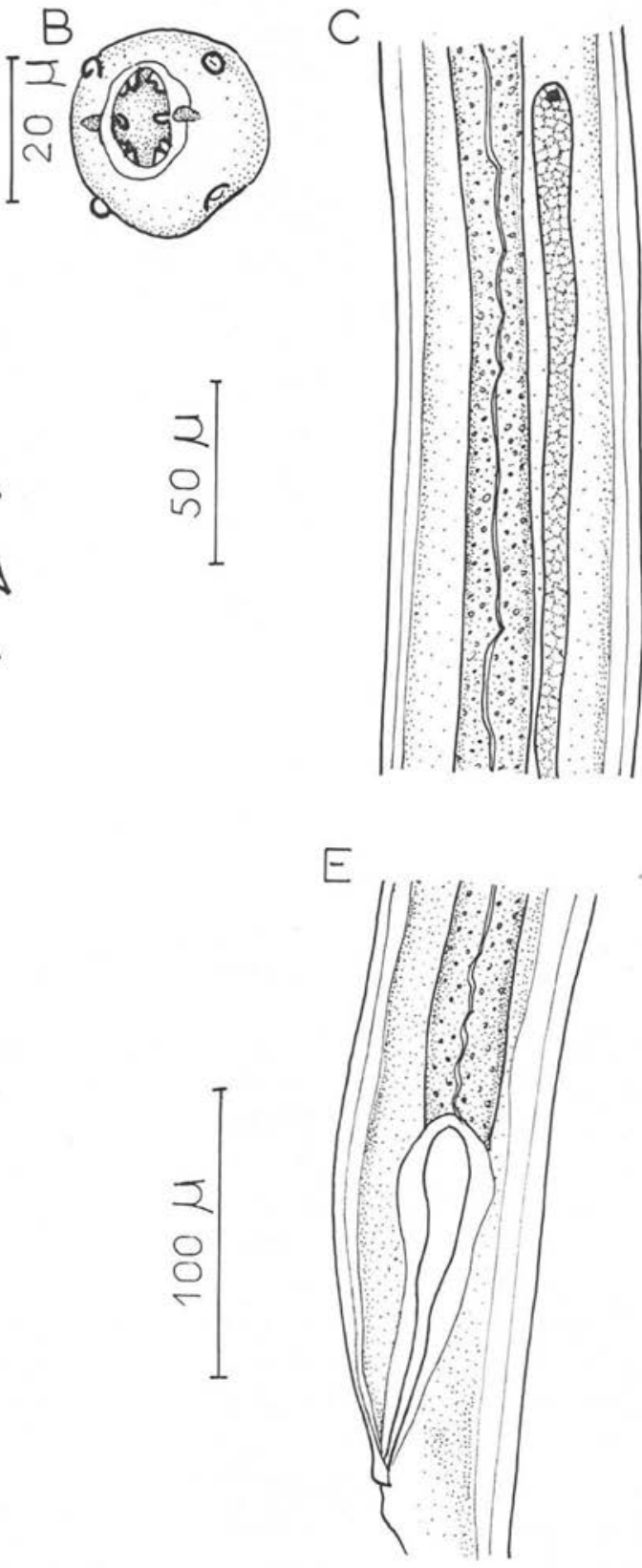

FIG. 2. - Spinitectoïdes berlandi Petter 1970, larve; A: extrémité antérieure, vue latérale ; B : extrémité antérieure, vue apicale ; C : région proximale de l'ébauche génitale mâle; D : extrémité caudale, vue latérale; E: larve mâle, région de l'anus, vue latérale. A: échelle $50 \mu$; B : échelle $20 \mu ;$ C, D, E : échelle $100 \mu$ 
caudale du mâle avec ses 4 paires de papilles pré-cloacales groupées 2 par 2, ne la rapproche pas des Rhabdochona, mais au contraire du genre Ascarophis; nous maintenons donc l'espèce dans la sous-famille des Cystidicolinae; dans cette sous-famille, si l'on excepte l'extrémité apicale qui est très voisine de celle de Cystidicola, l'ensemble des caractères: nombre des papilles pré-cloacales, longueur du pharynx, localisation intestinale, la rapproche plutôt des Ascarophis (voir Dollfus et Campana-Rouget 1956).

Il est intéressant de constater qu'à 2 reprises nous avons trouvé des adultes de cette espèce chez Molva molva, qui est donc l'hôte définitif spécifique, et des larves du $4^{\circ}$ stade chez Gadus luscus; cela semble indiquer que chez cet hôte, la $4^{\circ}$ mue ne peut avoir lieu et que les larves restent bloquées au $4^{\circ}$ stade.

\section{Cristitectus congeri n. g., n. sp.}

Dans l'estomac d'un Conger vulgaris Cuv., nous avons trouvé une femelle de Spiruride qui possède des caractères céphaliques très particuliers nécessitant la création d'un genre nouveau.

\section{Description :}

L'extrémité antérieure est en effet ornée de courtes arêtes cuticulaires disposées en cercle tout autour du corps (fig. 3, A) ; ces côtes sont de longueurs inégales, mais e!les se terminent toutes à $90 \mu$ de l'apex, un peu en arrière de la fin du pharynx ; en section transversale, on voit qu'elles sont réparties suivant un plan de symétrie bilatérale (fig. 3, B) : on observe 2 côtes médianes simples, 2 côtes latérales doubles, et 4 côtes submédianes également doubles. En dessous des côtes, la cuticule est légèrement dilatée sur une longueur de $250 \mu$.

L'ouverture buccale (fig. 3, B) est allongée dorso-ventralement et entourée de 2 pseudo-lèvres à bord ondulé; au milieu de chaque pseudo-lèvre, se trouve une forte dent latérale; de chaque côté de la dent, le bord de la pseudo-lèvre présente un épaississement chitinoïde formant une petite dent; il existe 4 papilles submédianes et 2 amphides portées par les pseudo-lèvres; intérieurement aux papilles submédianes, on observe 4 petites papilles médio-médianes.

Le pharynx (fig. 3, A) est court et élargi latéralement à sa partie antérieure ; l'œsophage est nettement divisé en un œsophage musculaire court et étroit et un œsophage glandulaire très long et de grand diamètre; le pore excréteur est légèrement en dessous de l'anneau nerveux; les deirides ont la forme de minuscules épines.

La queue, arrondie, porte 4 petites pointes à son extrémité (fig. 3, C).

La vulve est située à peu près à la limite entre le $2^{\circ}$ et le $3^{\circ}$ tiers du corps ; l'ovéjecteur, très court, (fig. 3, D), se divise en deux utérus opposés; les œufs, embryonnés au moment de la ponte, sont dépourvus de filaments polaires (fig. 3, E).

La femelle mesure $24 \mathrm{~mm}$; ses principales mensurations sont les suivantes: largeur maximum : $270 \mu$; pharynx: $70 \mu$; œsophage musculaire: $680 \mu$; œsophage 

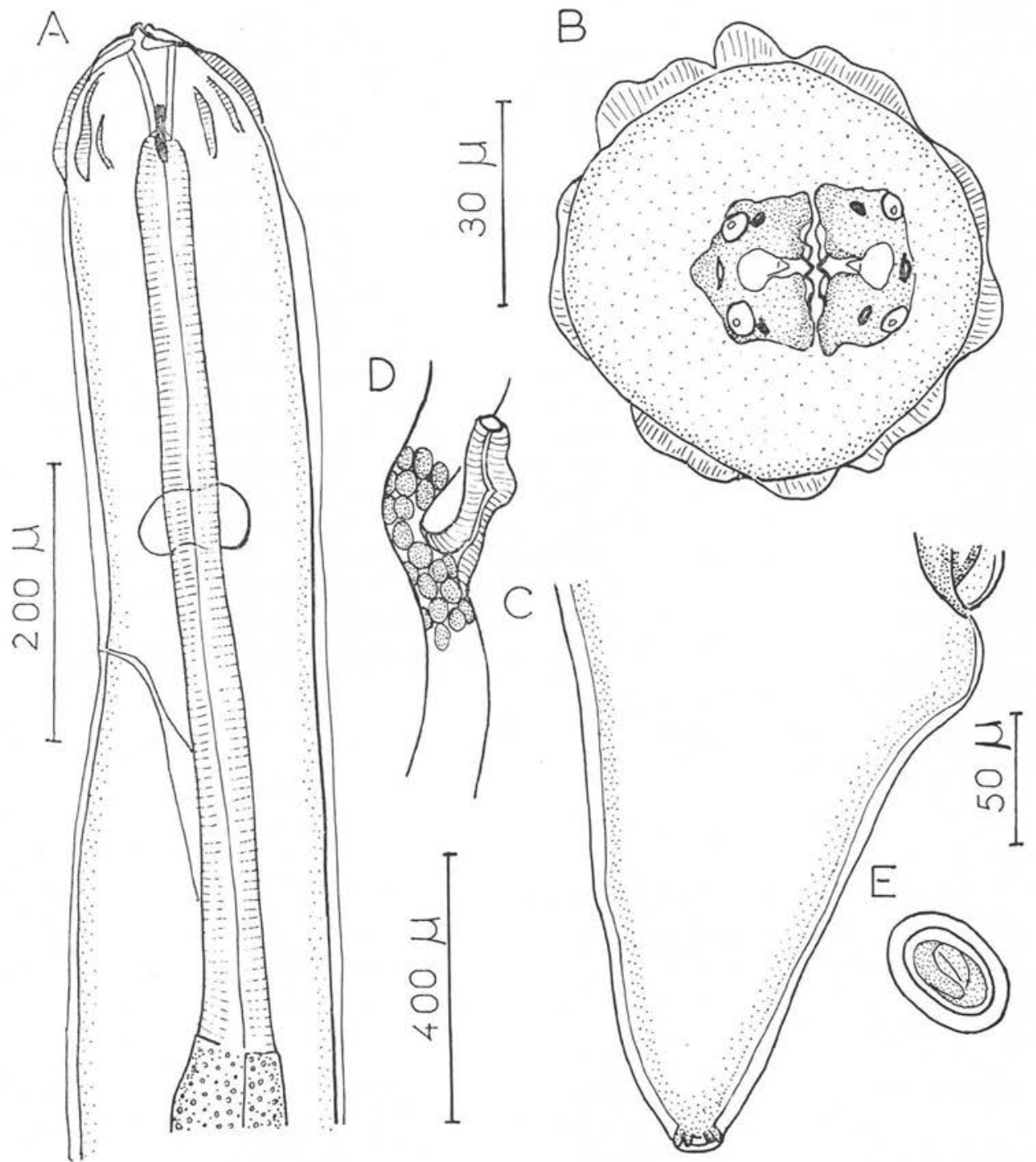

Fig. 3. - Cristitectus congeri n. sp. ; A : extrémité antérieure, vue latérale; B : extrémité antérieure, vue apicale; C: extrémité caudale, vue latérale; D: ovéjecteur; E: œuf. A : échelle $200 \mu$; B : échelle $30 \mu ; C$, E: éch:lle $50 \mu ; \mathrm{D}$ : échelle $400 \mu$

glandulaire : $4.950 \mu$; deirides, pore excréteur, anneau nerveux et vulve situés respectivement à $270 \mu, 450 \mu, 350 \mu$ et $15,7 \mathrm{~mm}$ de l'extrémité antérieure ; queue : $200 \mu$; œufs : $50 \mu$ sur $35 \mu$. 


\section{Discussion :}

Par la présence des côtes cuticulaires céphaliques et la structure apicale, cette espèce s'écarte de tous les genres connus chez les Spirurides de Poissons et nécessite la création d'un genre nouveau.

Bien qu'elle possède 8 papilles au cycle externe, l'espèce s'écarte complètement des Rhabdochoninae par la présence des pseudo-lèvres. L'existence de ces pseudo-levres la place plutôt dans les Cystidicolinae; cependant tous les Cystidicolinae connus n'ont que 4 papilles au cycle externe et des pseudo-lèvres réduites à un lobe médian. Les huit papilles du cycle externe et les pseudo-lèvres très développées, à bords ondulés et munis d'épaississements chitinoïdes, la rapprocheraient plutôt des Habronematinae, bien que, comme les Acuariidae, elle ne possède pas de lèvres médianes. Nous l'interprétons comme un Cystidicolinae plus primitif que les autres genres de la sous-famille, la réduction des pseudo-lèvres au lobe médian étant considérée comme un caractère évolué. Nous nommons ce genre Cristitectus n. g.

\section{Diagnose :}

Cystidicolinae: Bouche munie de deux pseudo-lèvres très développées, à bords ondulés et pourvus d'épaississements chitinoïdes; chaque pseudo-lèvre porte en son milieu une forte dent latérale; huit papilles au cycle externe; extrémité antérieure munie de courtes côtes chitinoïdes disposées en cercle autour du corps. Parasites du tube digestif des Poissons.

Espèce-type : Cristitectus congeri n. sp.

\section{Ascarophis morrbuae Van Beneden 1870}

Nous avons trouvé respectivement chez Gadus luscus L. trois femelles adultes et chez un Crabe juvénile de l'espèce Carcinus maenas Penn. deux larves de Spirurides que nous avons attribuées à l'espèce Ascarophis morrhuae.

\section{Femel.Les adultes :}

\section{Description :}

Les trois femelles adultes ont été trouvées dans l'estomac d'un Gadus luscus. Elles mesurent $14 \mathrm{~mm}$. La vue apicale est difficile à étudier en raison de sa petite taille, nous avons pu cependant observer une ouverture buccale losangique munie de deux grosses dents latérales (fig. 4, B), quatre papilles submédianes et deux amphides.

Le pharynx est long et élargi latéralement à sa partie antérieure (fig. 4, A), les stries cuticulaires prennent un aspect « en dent de scie » légèrement en dessous du pore excréteur, et cet aspect disparaît complètement un peu en avant de la vulve. La queue, courte et arrondie, se termine par un petit bouton (fig. 4, D). Deux femelles 
sur les trois examinées possèdent des œufs embryonnés; ces œufs possèdent à l'un des pôles, deux filaments bien nets; en plus de ces filaments, on observe à chacun des pôles des filaments beaucoup plus fins et dont la présence et le nombre sont variables (fig. 4, E, F).

Les principales dimensions sont les suivantes: longueur: $14 \mathrm{~mm}$; largeur: $150 \mu$; pharynx : $145 \mu$; œsophage musculaire : $510 \mu$; œsophage glandulaire : $2150 \mu$;

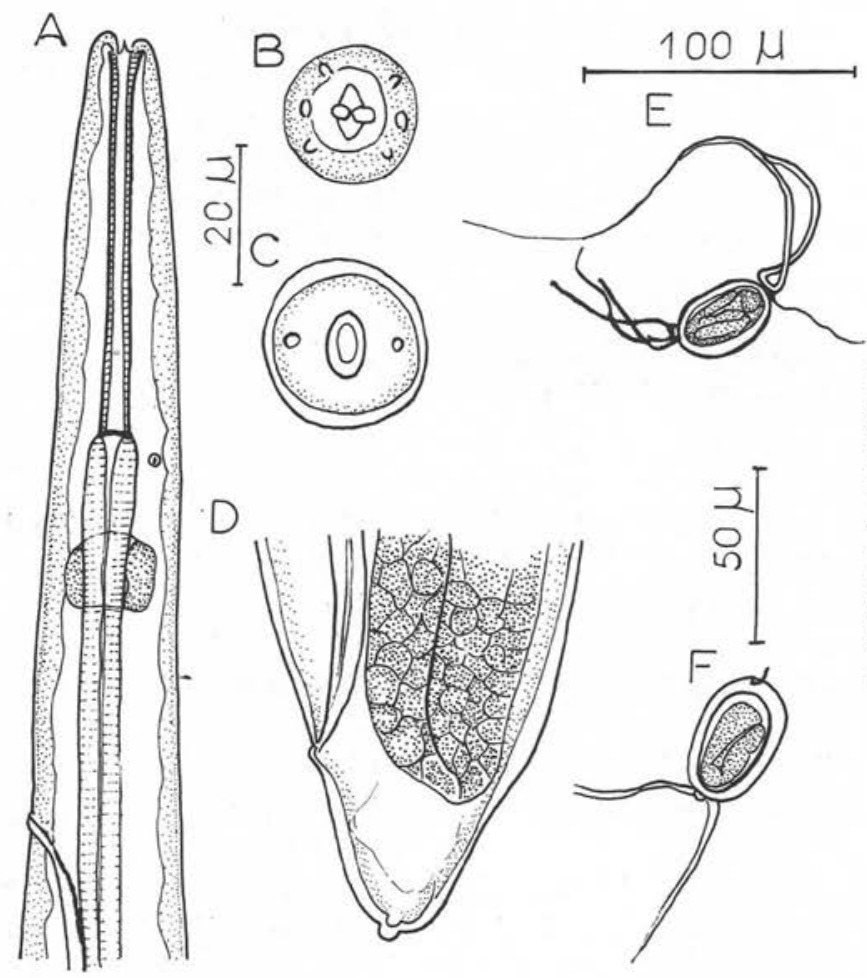

Fig. 4. - Ascarophis morrhuae van Beneden 1870 ; A : extrémité antérieure, vue latérale; B: extrémité antéri ure, vue apicale superficielle ; C: extrémité antérieure, vue apica:e, coupe op:ique; D : extrémité caudale, vue latérale; $\mathrm{E}, \mathrm{F}$ : œufs. A, D, E: échelle 170 u; B, C: échelle $20 \mu$; F : échelle $50 \mathrm{u}$

queue : $70 \mu$; deirides, pore excréteur, anneau nerveux et vulve situés respectivement à $150 \mu, 280 \mu, 190 \mu$ et $8,3 \mathrm{~mm}$ de l'extrémité antérieure ; œufs : $38 \mu$ sur $28 \mu$.

\section{Diseussion :}

Par leurs dimensions et tous leurs caractères, ces femelles correspondent parfaitement aux femelles décrites par Baylis en 1933 chez Trigla lineata et qu'il attribue à Ascarophis morrhuae; Baylis n'a pas observé de filaments polaires, mais ses œufs ne contenaient pas d'embryons complètement formés et n'étaient donc pas mûrs. 
Dollfus et Campana-Rouget (1956) estiment que l'espèce de Baylis n'est pas Ascarophis morrhuae, car «les descriptions récentes d'Ascarophis de Gadidés montrent des différences assez sensibles avec celle de Baylis, surtout en ce qui concerne la taille»; ils nomment les spécimens de Baylis, Ascarophis baylisi; cependant Rahman (1966), après avoir réexaminé les spécimens de Baylis, conclut que Ascarophis baylisi est synonyme d'Ascarophis morrhuae, la taille des spécimens entrant dans les intervalles de tailles donnés par Punt pour Ascarophis morrhuae. Le fait que nous retrouvions des spécimens tout à fait identiques à ceux de Baylis chez un Gadus luscus et non chez un Trigla est un nouvel argument en faveur de la conclusion de Rahman.

La plupart des auteurs considèrent la présence et le nombre des filaments polaires sur les œufs comme un caractère spécifique ; nous voyons d'après nos propres observations que ce caractère doit être considéré avec méfiance, puisque chez nos spécimens des filaments plus fins en nombre variable s'ajoutent aux filaments principaux; une diagnose basée sur les œufs devrait en tout cas ne considérer que des œufs prêts à être pondus.

Larves: A l'autopsie d'un crabe juvénile de l'espèce Carcinus maenas Penn. (1), nous avons recueilli deux larves de Spiruride:

\section{Description :}

L'extrémité antérieure est munie de dents latérales, le pharynx est long et élargi latéralement à son extrémité antérieure (fig. 5, A), l'œsophage musculaire et l'œsophage glandulaire sont bien délimités; la queue se termine par un petit bouton arrondi (fig. 5, C), la cuticule présente un aspect en dent de scie qui débute en dessous de l'anneau nerveux et s'étend sur une longueur de $950 \mu$ en dessous de la fin de l'œsophage glandulaire (fig. 5, B).

Chez la larve mâle, l'ébauche génitale a la forme d'un long cordon impair, chez la larve femelle, elle se présente sous forme de deux cordons opposés reliés par un long tube très étroit.

Principales dimensions: longueur: $4,1 \mathrm{~mm}$; largeur: $50 \mu$; pharynx: $120 \mu$; œsophage musculaire : $300 \mu$; œsophage glandulaire: $950 \mu$; queue : $50 \mu$; deırides, pore excréteur et anneau nerveux situés respectivement à $120 \mu, 210 \mu$ et $150 \mu$ de l'extrémité antérieure.

\section{Discussion :}

Les proportions des différents organes et l'ornementation en dent de scie de la cuticule nous font supposer que ces larves appartiennent à l'espèce Ascarophis morrhuae.

Les larves de cette espèce ont déjà été signalées et décrites, en particulier par Uspenskaïa (1953); celle-ci décrit des larves de plus petite taille, et dont l'œsophage glandulaire en particulier est nettement plus court. Uspenskaïa (1953) signale que dans

(1) Nous remercions bien vivement $\mathrm{M}^{\mathrm{me}}$ Grmek-Guinot de nous avoir déterminé ce spécimen. 
la mer de Barents l'hôte le plus habituel des larves est Eupagurus pubescens $(\mathrm{H}$. Kröyer 1838), mais qu'on trouve également ces larves chez les hôtes suivants : Hetairus polaris

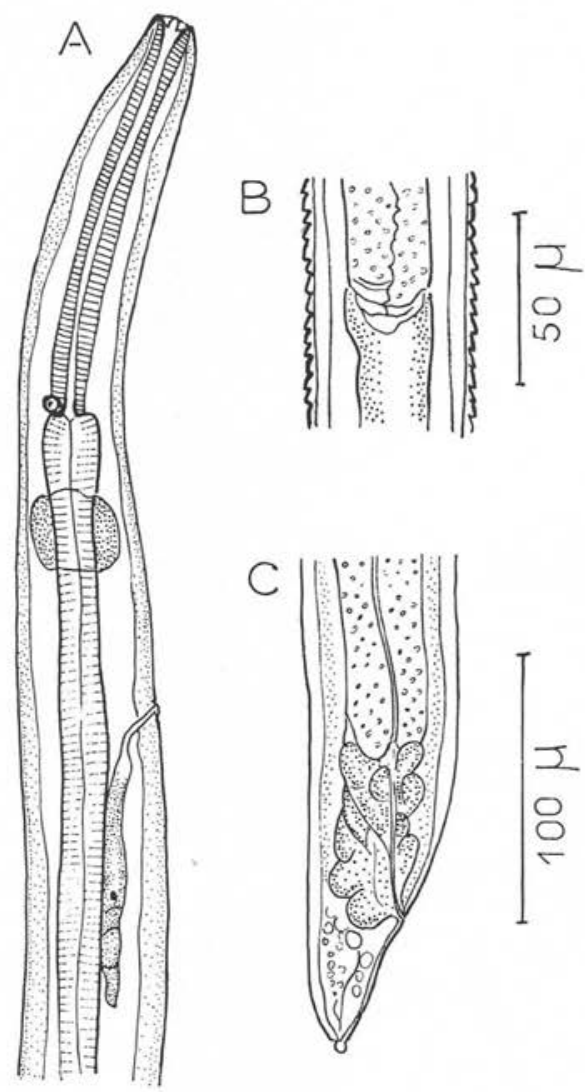

Fig. 5. - Ascarophis morrhuae, larve; A : extrémité antérieure, vue latérale; $\mathbf{B}$ : région de la jonction œsophago-intestinale, montrant la cuticule en dent de scie; C: extrémité caudale, vue latérale. A, B: échelle $50 \mathrm{u} ; \mathrm{C}$ : échelle $100 \mu$

(Sabine 1824), Spirontocaris spinus (James Sowerby 1806) et Pandalus borealis H. Kröyer 1838. Carcinus maenas doit donc être ajouté à la liste des hôtes intermédiaires où les larves d'Ascarophis morrhuae ont été trouvées.

\section{Mâle d'Ascarophis sp.}

Dans l'estomac d'un Trigla sp., nous avons trouvé des débris de nourriture parmi lesquels un crabe encore entier (2); celui-ci a été écrasé dans l'eau et nous avons récolté dans les eaux de rinçage un mâle de Spiruride adulte dont nous donnons la description :

(2) Nous n'avons malheureusement pas déterminé l'espèce à laquelle appartient ce crabe. 

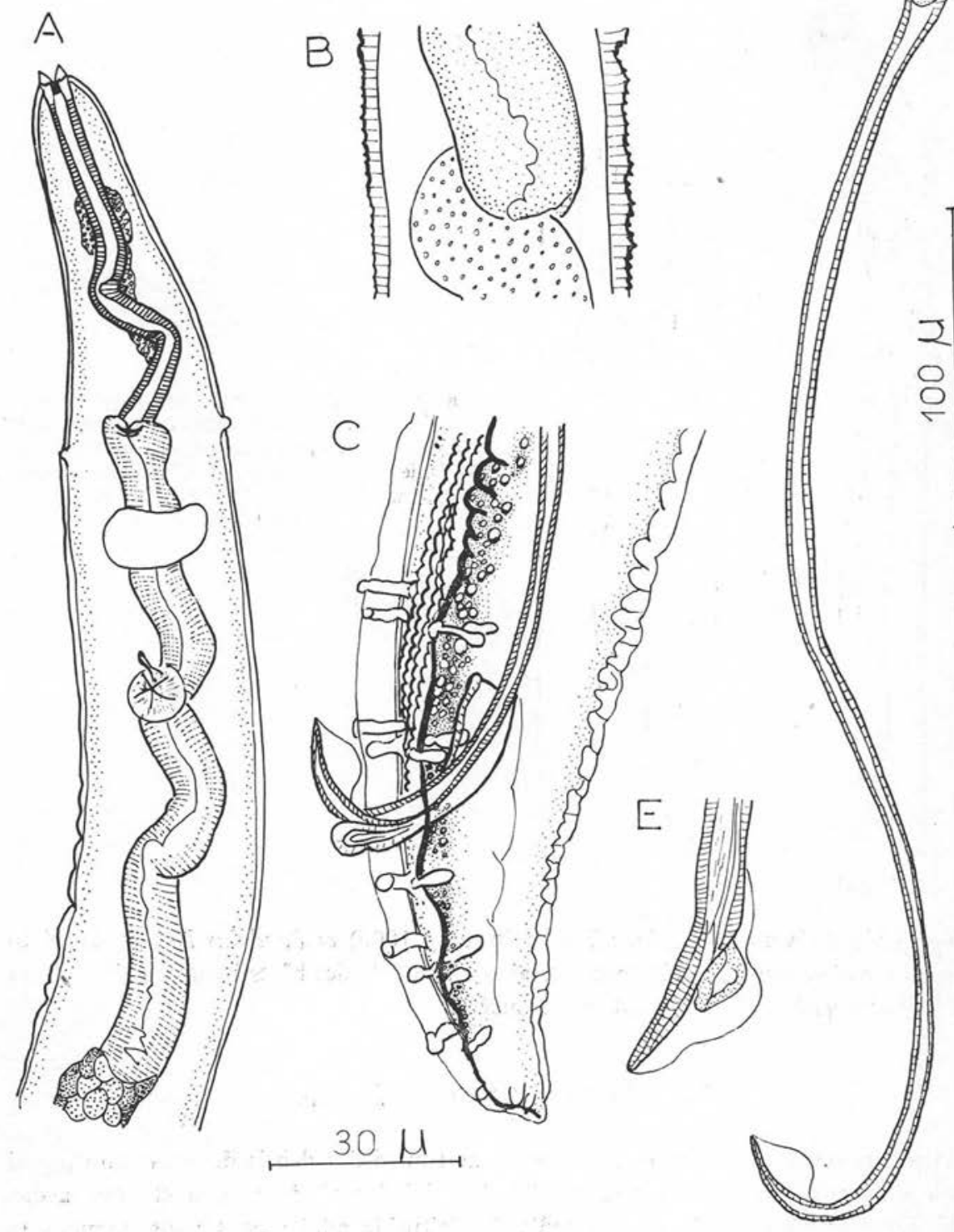

Fig. 6. - Mâle d'Ascarophis sp.; A : extrémité antérieure, vue médiane; B : région de la jonction œesophago-intestinale; C: extrémité caudale, vue latéro-médiane; D: grand spicule; E: grand spicule, extrémité distale. A, B, C, D : échelle $100 \mu ; \mathrm{E}$ : échelle $30 \mu$ 


\section{Description :}

Il mesure $5,45 \mathrm{~mm}$; la tête porte deux fortes dents latérales dressées ; le pharynx est très long (fig. 6, A) ; l'œsophage musculaire et l'œsophage glandulaire sont bien délimités; la queue du mâle (fig. $6, \mathrm{C}$ ) porte quatre paires de papilles pré-cloacales groupées 2 à 2 et cinq paires de papilles post-cloacales disposées comme l'indique la figure; les phasmides sont en-dessous de la dernière paire; le spicule gauche est très long et effilé, son extrémité distale est élargie par la présence d'une aile membraneuse (fig. 6, D et E) ; le spicule droit est court et trapu, avec une extrémité distale arrondie.

Il existe d'étroites ailes caudales ornées de tubercules qui s'étendent depuis l'avant-dernière paire de papilles cloacales jusqu'à $60 \mu$ au-dessus de la première paire pré-cloacale. La face ventrale au-dessus du cloaque est ornée sur une longueur de $225 \mu$ de stries longitudinales.

Les stries cuticulaires se chevauchent légèrement, présentant en section un aspect en très fines dents de scie, qui débute à $240 \mu$ de l'extrémité antérieure et se termine à $2.100 \mu$ de l'extrémité postérieure (fig. 6, B).

Principales dimensions: longueur : 5,45 mm; largeur: $75 \mu$; pharynx : $130 \mu$; œsophage musculaire : $230 \mu$; œsophage glandulaire: $1.350 \mu$; queue : $110 \mu$; spicule gauche : $480 \mu$; spicule droit : $80 \mu$; deirides, pore excréteur et anneau nerveux situés respectivement à $125 \mu, 160 \mu$ et $180 \mu$ de l'extrémité antérieure.

\section{Discussion :}

Ce spécimen présente tous les caractères des mâles d'Ascarophis; l'ensemble des dimensions et la disposition des papilles cloacales correspondent bien au mâle d'Ascarophis morrhuae décrit par Gordon (1951) et Poljanski (1952).

Cependant, la striation cuticulaire «en dents de scie » nous paraît peu accentuée par rapport à celle qui est décrite chez Ascarophis morrhuae et que nous avons observée nous-mêmes sur les larves de cette espèce décrites ci-dessus; de plus, deux caractères de notre spécimen ne sont pas signalés chez le mâle d'Ascarophis morrhuae : les tubercules qui ornent les ailes caudales et l'extrémité élargie et ailée du spicule gauche ; cependant, l'extrémité du spicule est difficile à observer quand il ne fait pas saillie hors du cloaque ; quant à l'ornementation des ailes caudales, elle est décrite chez deux espèces d'Ascarophis, Ascarophis filiformis Poljanski, 1952 et Ascarophis pacificus Zhukov, 1960, qui diffèrent de notre spécimen par les caractères de la cuticule, et elle existe peut-être sans avoir été signalée chez la plupart des espèces ; en fait, il existe plusieurs espèces d'Ascarophis dont les mâles pourraient correspondre à notre spécimen, et nous préférons donc ne pas le nommer. 


\section{Larve d'Ascarophis sp.}

A l'autopsie d'un Acanthocottus bubalis (Euphr.) pêché dans des flaques à marée basse, nous avons trouvé deux larves de Spiruride qui, par leurs caractères céphaliques appartiennent vraisemblablement au genre Ascarophis.

\section{Description :}

Elles sont longues d'environ $9 \mathrm{~mm}$.

L'ouverture buccale est allongée dorso-ventralement et entourée par deux pseudolèvres latérales très développées, qui portent en leur milieu deux fortes dents côniques ; il existe quatre grosses papilles submédianes et deux amphides (fig. 7, B, C).

Le pharynx est court et élargi latéralement à son extrémité antérieure (fig. 7, A) ; l'œsophage musculaire et l'œsophage glandulaire sont bien délimités; la queue est courte et arrondie (fig. 7, D) ; l'ébauche génitale a la forme d'un long cordon.

Principales mensurations: longueur: $9 \mathrm{~mm}$; largeur: $100 \mu$; pharynx : $60 \mu$; œsophage musculaire: $465 \mu$; œsophage glandulaire: $2.200 \mu$; anneau nerveux et pore excréteur situés respectivement à $200 \mu$ et $290 \mu$ de l'extrémité antérieure ; queue : $110 \mu$; les deirides ne sont pas visibles.

\section{Diseussion :}

Nous ne pouvons évidemment déterminer de façon précise cette larve que nous supposons appartenir au genre Ascarophis : cependant, le pharynx très court l'éloigne de la plupart des espèces de ce genre ; les pseudo-lèvres très développées rappellent la structure apicale de l'espèce Cristitectus congeri $\mathrm{n}$. sp. que nous décrivons ci-dessus.

Signalons qu'une larve d'aspect assez comparable à la nôtre a été décrite en 1968 chez Homarus americanus par Uzmann (3).

\section{Bibliographie}

Berland (B.), 1961. - Nematodes from some Norwegian marine fishes. Sarsia. Bergen, 2, $1-50$.

Dollfus (R. Ph.) \& Campana-Rouget (Y.), 1956. - Une nouvelle espèce d'Ascarophis (Nematoda, Spirurinae) chez Gadus luscus L. Révision du genre. Ann. Parasit., 31, (4), 385-404.

Gordon (A. R.), 1951. - On the male of Ascarophis morrhuae Van Ben. Parasit., 41, (3-4), 261-263.

(3) Nous remercions vivement M. R.-Ph. Dollfus pour nous avoir communiqué sa bibliographie sur le genre Ascarophis. 

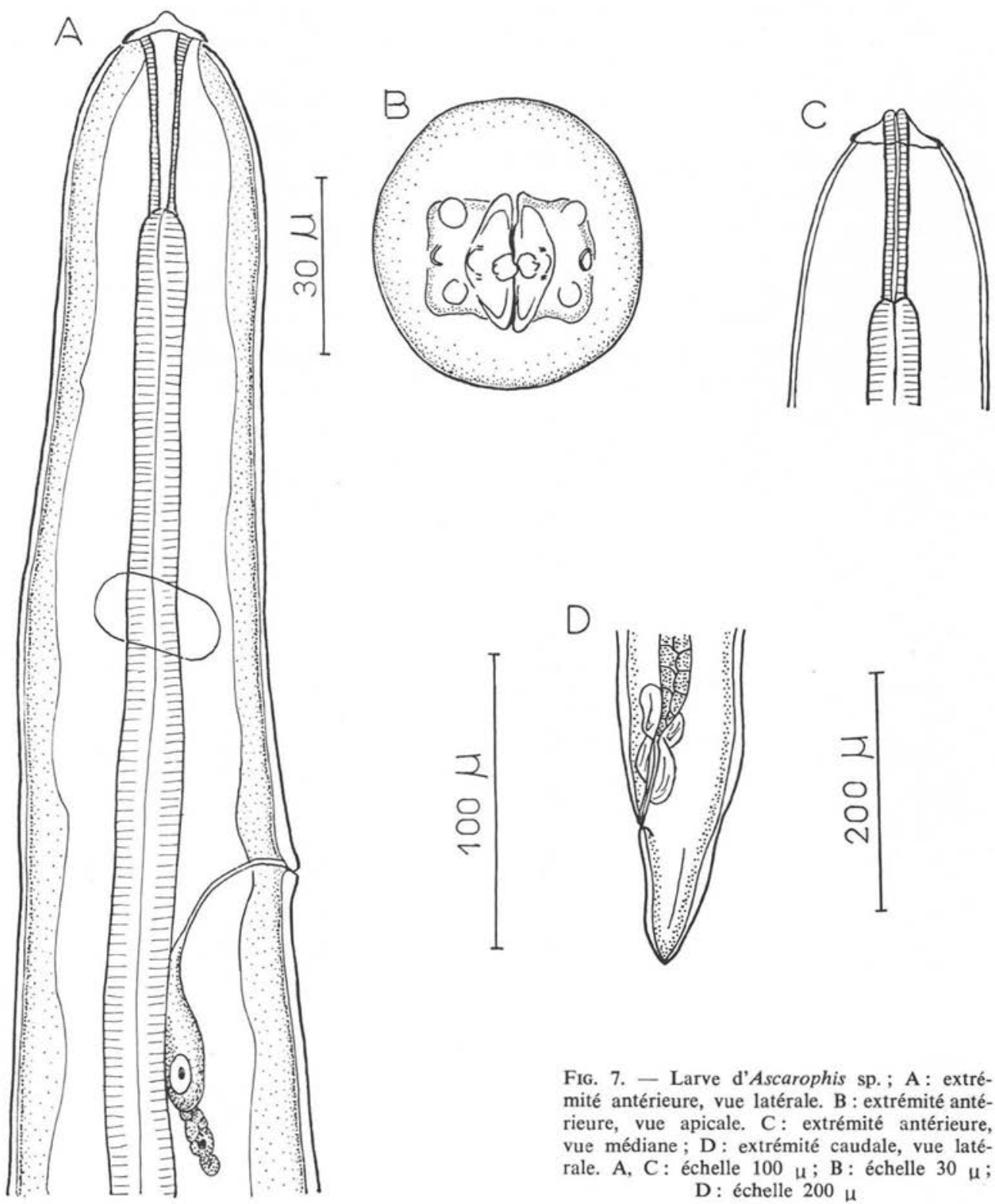

Fig. 7. - Larve d'Ascarophis sp.; A: extrémité antérieure, vue latérale. B : extrémité antérieure, vue apicale. C: extrémité antérieure, vue médiane; D: extrémité caudale, vue latérale. A, C: échelle $100 \mu$; B : échelle $30 \mu$; D : échelle $200 \mu$ 
Petter (A. J.), 1970. - Enquête sur les Nématodes de Poissons de la région nantaise ; identification des larves d'Ascarides parasitant les Sardines (en rapport avec les granulomes éosinophiles observés chez l'Homme dans la région). Ann. Parasit., 44 (5), 559-580.

PolJANSKI (G. I.), 1952. - Quelques Nématodes nouveaux et peu connus, parasites de l'intestin de Poissons marins (en Russe). - Trudi Zoologitch Inst. Moscou, 12, 133-147.

Rahman (H.), 1966. - Ascarophis crassicollis Dollfus and Campana-Rouget in Scottish waters. Ann. Mag. nat. Hist., Year 1965, Ser. XIII, 8 (87-88), 187-192.

UspensKaia (A. V.), 1953. - Le cycle évolutif des Nématodes du genre Ascarophis van Beneden (Nématodes: Spirurata). Zool. Zurn., 32 (5), 828-832.

Uzmann (J. R.), 1967. - Juvenile Ascarophis (Nematoda: Spiruroidea) in the American Lobster: Homarus americanus. J. Parasit., 53 (1), 218.

Zhuкov (E. V.), 1960. - Endoparasitic worms of the Fishes in the Sea of Japan and SouthKuril Shallow-waters. Tr. Zool. Inst. AN SSSR., 28, 3-146. 